\title{
Rhabdomyosarcoma in an adult with HIV
}

\author{
Sachin Jhawar, BS, ${ }^{1}$ Gaurav Sharma, $\mathrm{MD},{ }^{2}$ and Harold Ballard, $\mathrm{MD}^{3}$ \\ ${ }^{1}$ New York University School of Medicine, New York; ${ }^{2}$ Department of Medicine, LAC+USC Medical Center, Keck School of \\ Medicine, University of Southern California, Los Angeles, California; ${ }^{3}$ Medical Service, Department of Veterans Affairs Medical \\ Center, New York
}

$\mathrm{R}$ habdomyosarcomas are a rare group of soft tissue neoplasms of mesenchymal origin. RMS is common among childhood cancers, but it is among the rarest of adult tumors. They account for about $5 \%$ of all childhood cancers. ${ }^{1}$ Soft-tissue sarcomas account for less than $1 \%$ of adult malignancies, and RMS account for only $3 \%$ of those sarcomas. ${ }^{2}$ Here, we report a case of RMS in the neck, which led to dysphagia due to external compression of the esophagus.

\section{Case presentation}

A 62-year-old man presented with 1 month of progressive dysphagia and several days of dyspnea on exertion. The patient had been previously seen in the walk-in clinic for several weeks of sore throat, dysphagia, and cough. He was given 7 days of levofloxacin. One month after his clinic visit, he was seen for scheduled management of his HIV and HAART (highly active antiretroviral therapy), at which time his viral load was undetectable and CD4 count was 410 . He was referred to the GI clinic because of persistent dysphagia, cough, new-onset odynophagia, and unintentional weight loss. Gastroenterology prescribed an empiric treatment with fluconazole and follow-up after 1 week. The patient returned after 1 week without any improvement in symptoms. Esophagoduodenoscopy at that time revealed extrinsic compression in the cervical esophagus with $25 \%$ of the lumen occupied. Biopsy of a single plaque in the cervical esophagus had cytological features suggestive of herpes virus infection. The patient was recommended to undergo a computed tomography scan

Manuscript received September 10, 2012; accepted November 5, 2012.

Correspondence Sachin Jhawar, BS, New York University School of Medicine, 530 First Avenue, New York, NY 10010 (Sachin.Jhawar@nyumc.org).

Disclosures The authors have no conflicts of interest to disclosures. of the head and neck and to return after 3 weeks. The patient failed to comply and presented 1 month later because of continued cough, dysphagia, odynophagia, and a 20-25 pound unintentional weight loss. He reported cough productive of yellow sputum, inability to swallow solid food, but no difficulty with liquids. He also reported 3 days of chills, night sweats, and dyspnea on exertion with reduced exercise tolerance.

The patient reported intermittent compliance with his HAART over the previous month due to his dysphagia. His HIV was highly resistant and included the following salvage therapy: abacavir SO4 $600 \mathrm{mg} / \mathrm{lamivudine} 300 \mathrm{mg}$, darunavir 300 $\mathrm{mg}$, raltegravir $400 \mathrm{mg}$, and ritonavir $100 \mathrm{mg}$. He also had a history of untreated hepatitis $\mathrm{C}$ and opportunistic infections, which included oral candidiasis and pneumocystis carinii pneumonia (PCP). His exposures included extensive substance abuse (IV heroin, cocaine, marijuana, alcohol) and unprotected sex with several partners. The patient also reported chronic obstructive pulmonary disease. He had a 20 pack-year smoking history.

On presentation to the emergency department, the patient was in moderate distress from coughing, able to speak full sentences, but appearing acutely ill. He was febrile to $101.2^{\circ} \mathrm{F}$, in sinus tachycardia at $108 \mathrm{bpm}$, with blood pressure of $124 / 85$, and breathing 14 times per minute with $85 \%$ oxygen saturation with exertion and $90 \%$ at rest. The patient's physical exam was notable only for a prominent $1-2 \mathrm{~cm}$ right cervical lymph node and faint lung crackles bilaterally.

Laboratory values were notable for an elevated lactic acid dehydrogenase level of $\mathrm{LDH}$ of $459 \mathrm{U} / \mathrm{L}$ (normal, 0-250) and a mildly elevated aspartate transaminase of $61 \mathrm{U} / \mathrm{L}$ (normal, 0-42). Chest radiography revealed diffusely increased

Commun Oncol 2013;10:175-177 DOI: 10.12788/i.cmonc.0041 
interstitial markings throughout both lungs and small bilateral effusions, right greater than left. The results of the CT scan of the neck with contrast showed a large esophageal mass suspicious for primary neoplasm and an enlarged left infrahyoid neck lymph node compatible with metastatic lymphadenopathy. A fine-needle aspiration of the prominent left cervical lymph node showed a poorly differentiated malignant neoplasm. Follow-up core biopsy of the same lymph node was significant for a pleiomorphic rhabdomyosarcoma. The patient was subsequently started on palliative chemotherapy: cyclophosphamide (D1), doxorubicin (D1, 2, 3), vincristine (D1), ifosfamide, and etoposide (D22, 23, 24, 25, 26) as part of a 42-day cycle. Cytopathology results of the increasing right pleural effusion showed large anaplastic malignant cells consistent with the diagnosis of rhabdomyosarcoma.

The patient's hospital course was complicated by alterations in mental status, dyspnea, and the development of infectious ulcers on his buttocks and scrotum. After 2 cycles of chemotherapy, the esophageal mass demonstrated some decrease in size, but the patient's condition continued to deteriorate. The patient eventually passed away after he decided to forego chemotherapy.

\section{Discussion}

It often incorrectly thought that RMS can only occur in striated muscles. In fact, they can arise in other tissues, including the head and neck region, which accounts for $40 \%$ of cases, the nonprostate genitourinary region $(15 \%$ of cases), bladder and prostate tumors (10\%), the limbs, and other sites, including the thorax and abdomen (20\%). ${ }^{3}$ Fewer than $10 \%$ of cases of RMS are found in the neck. ${ }^{4}$ In such cases, presenting symptoms are usually changes in speech, swallowing, or respiratory symptoms.

There are 3 major histological subtypes - embryonal, alveolar, and pleomorphic. Although pleomorphic RMS accounts for less than $1 \%$ of all pediatric cases of the disease, it is by far the most common entity in the adult population. Pleomorphic RMS is extremely aggressive and is thought to be closer to high-grade adult soft-tissue sarcoma than pediatric RMS. This, along with the fact that adults tend to present at later stages, may partly account for why adults tend to do more poorly with RMS than do children. ${ }^{3}$ Treatment is multidisciplinary (surgery, radiotherapy, and chemotherapy). In as much as it tends to behave like adult soft-tissue sarcomas, pleomorphic RMS may be more amenable to adjuvant chemotherapy than the other subtypes. ${ }^{5}$

Patients with HIV have historically had an increased incidence of malignancy. Early on in the epidemic, before the advent of adequate antiretroviral therapy, there was a higher propensity of "AIDS-defining malignancies." including Kaposi's sarcoma, cervical cancer, and nonHodgkin lymphoma. ${ }^{6,7}$ What makes these 3 malignancies "AIDS defining" is that there is a direct correlation with the immunologic decline and the incidence of these particular cancers. Although the HIV virus is not considered to be pathogenic, it is thought to facilitate malignant transformation via several mechanisms, particularly its immunosuppressive role. ${ }^{8}$

HAART changed the landscape of malignancy associated with HIV. There has been a marked difference in the incidence of certain non-AIDS defining cancers (NADCs) since the advent of HAART, contributing to a significant amount of mortality in AIDS patients. Immunodeficiency is not a prerequisite to these NADCs. Therefore, while HAART therapy uniformly may decrease the incidence of Kaposi's sarcoma and nonHodgkin lymphoma, ${ }^{9}$ it does not uniformly decrease the risk of NADC. A review by Pantanowitz and Dezube discussed several risk factors for NADC, including length of immunosuppression, age over 40 years, coinfection with oncogenic viruses, familial cancer history, microsatellite alterations, and lifestyle habits, including smoking, sexual practices, and sun exposure. ${ }^{10}$

Included in the category of NADC, have been soft tissue sarcomas. While leiomyosarcomas ${ }^{11}$ and Kaposi's sarcoma have been found to occur at an increased rate in AIDS patients, particularly those with a low CD4 counts, there have been very few case reports of other soft-tissue malignancies, including cardiac angiosarcoma. There have, however, been very few case reports of adult onset RMS in AIDS patients. Although there have been a few case reports of rhabdomyosarcoma in adults at other anatomic sites, ${ }^{12}$ to our knowledge, the present case is the first reported case of pleomorphic RMS in the neck in an AIDS patient.

\section{References}

1. McCarville MB, Spunt SL, Pappo AS. Rhabdomyosarcoma in pediatric patients: the good, the bad, and the unusual. $A m J$ Roentgenol. 2001;176(6): 1563-1569.

2. Weiss S, Goldblum JR. Rhabdomyosarcoma, in Enzinger and Weiss's Soft Tissue Tumors, in Weiss and S Goldblum, eds. JR 2001, Mosby: St. Louis, MO. p. 785-835.

3. Van Rijn RR, Wilde JC, Bras J, Oldenburger F, McHugh KM, Merks JH. Imaging findings in noncraniofacial childhood rhabdomyosarcoma. Pediatr Radiol. 2008;38(6):617-634.

4. Dagher R, Helman L. Rhabdomyosarcoma: an overview. Oncologist. 1999;4(1):34-44.

5. Frustaci S, Gherlinzoni F, De Paoli A, et al. Adjuvant chemotherapy for adult soft tissue sarcomas of the extremities and girdles: results of the Italian randomized cooperative trial. J Clin Oncol. 2001;19(5):1238-47.

6. Engels EA, Rosenberg PS, Frisch M, Goedert JJ. Cancers associated with Kaposi's sarcoma (KS) in AIDS: a link between KS herpesvirus and immunoblastic lymphoma. Br J Cancer. 2001; 85(9):1298-303.

7. Robinson W. Invasive and preinvasive cervical neoplasia in human immunodeficiency virus-infected women. Semin Oncol. 2000; 27(4):463-470. 
8. Carbone A, Gloghini A. AIDS-related lymphomas: from pathogenesis to pathology. Br J Haematol. 2005;130(5):662670.

9. Clifford GM, Polesel J, Rickenbach M, et al. Cancer risk in the Swiss HIV Cohort Study: associations with immunodeficiency, smoking, and highly active antiretroviral therapy. J Natl Cancer Inst. 2005;97(6):425-432.
10. Pantanowitz L, Dezube BJ. Evolving spectrum and incidence of nonAIDS-defining malignancies. Curr Opin HIV AIDS. 2009;4(1):27-34.

11. Jenson HB. Review: Epstein-Barr virus-associated smooth muscle tumors. Clin Adv Hematol Oncol. 2010;8(6):426-428.

12. Lauretti L, Montano N, Paternoster G, et al. Huge cranio-cerebral rhabdomyosarcoma in HIV-positive patient. J Neurooncol. 2010; 100(1):153-155. 